\title{
One-year clinical evaluation of e.max CAD and LavaTM ultimate laminate veneers with butt joint preparation (Randomized controlled clinical trial)
}

\author{
Mai M ElGendi ${ }^{*}$, Karim Rabie ${ }^{2}$ and Amina A Zaki ${ }^{3}$ \\ ${ }^{1}$ Dentist at Ministry of Health, Egypt \\ ${ }^{2}$ Lecturer of Fixed Prosthodontics, Faculty of Dentistry, Cairo University \\ ${ }^{3}$ Professor of Fixed Prosthodontics, Faculty of Dentistry, Cairo University
}

\begin{abstract}
Purpose: The aim of this clinical study was to evaluate ceramic laminate veneers constructed from Lithium disilicate (IPS e.max CAD), and resin nano-ceramic (Lava Ultimate ${ }^{\mathrm{TM}} \mathrm{CAD} / \mathrm{CAM}$ ) according to the modified United States of Public Health Service (USPHS) criteria over 12 months.

Materials and methods: Twenty-eight ceramic laminate veneers (fourteen for each group) were placed in the Fixed Prosthodontics Department clinics, Faculty of Dentistry, Cairo University by one operator. The restorations were made using CEREC premium 4.4 CAD/CAM software with IPS e.max CAD and Lava Ultimate $^{\mathrm{TM}} \mathrm{CAD} / \mathrm{CAM}$ blocks. Modified USPHS criteria were used to evaluate the clinical performance of the restorations. Patient satisfaction were assessed using visual analogue scale. Fisher's Exact test was used to compare between the two groups. Friedman's test was used to study the changes by time in each group. Patient satisfaction scores (VAS scores) were presented as median and range values.

Results: Regrading marginal adaptation, retention, and fracture, through the whole study period; all restorations in the two groups showed (Alpha) scores. While for marginal discoloration, all surfaces of restorations in the two groups showed (Alpha) scores till 6 months. While after 8,10 and 12 months; the palatal surface of Lava Ultimate group showed (Bravo) scores. However, there was no statistically significant difference between the two groups after 12 months $(P$-value $=0.481)$. The overall satisfaction of restorations was very satisfied by $85.7 \%$ and satisfied by $14.3 \%$.
\end{abstract}

Conclusion: Both IPS e.max CAD and Lava Ultimate laminate veneers revealed high successful clinical performance over a period of 12 months follow up.

\section{Introduction}

The treatment of defective and discolored anterior dentitions has always created a challenge in dentistry. Ceramic laminate veneers offer a conservative esthetic treatment, which met patient expectations for many years [1].

Various common techniques to fabricate porcelain veneers include the platinum foil or refractory die technique, in which porcelain in powder form is stacked on top of a refractory die and then fired in the oven. Another method is the pressing ceramic technique involving waxing up the restoration to the proper form and pressing it under pressure into the evacuated mold, similar to the lost wax technique for metal. The most advanced technique uses the Computer-aided Designing/Computer-aided Manufacturing "CAD/ CAM" system [2].

Two main types of materials are currently available for esthetic CAD/CAM-processed indirect dental restorations: glass-ceramics and hybrid resin ceramics [3].

The use of IPS e.max CAD is well documented in the literatures having successful restoration modality, due to its superior optical and mechanical properties. The introduction of hybrid ceramic materials combined the advantages of both glass ceramics and composite resin. Resin nano-ceramic is a mixture of a resin composite matrix and nanoceramic fillers of approximately $80 \%$ by weight [4].
In a direct comparison between properties, glass-ceramic materials are superior to resin-composites. The attractiveness of the latter is based on ease of fabrication and the possibility of an easier and more feasible intra-oral repair of minor defects induced by function [3].

Therefore, combination of both material properties in one material resulted in characteristic superior properties [3].

Dentists should base their choice of material on the requirements of the tooth being restored, such as the indication and the necessity of the tooth preparation to improve esthetics and function.

Clinical studies for comparative evaluation of both CAD/CAM glass ceramic and hybrid ceramic laminate veneers is absent in the literature and more attention should be drawn to clinical comparison of both materials.

Hypothesis of this study that there will be no difference in the clinical performance of Lava Ultimate if compared to IPS e.max CAD ceramic laminate veneers for restoration of teeth in esthetic zone.

${ }^{\star}$ Correspondence to: Mai M ElGendi, Dentist at ministry of health, Cairo, Egypt, E-mail: mai.eltohami@dentistry.cu.edu.eg

Key words: clinical evaluation, clinical performance, patient satisfaction, laminate veneers, ips e.max, lithium disilicate, lava ultimate, resin nano ceramics

Received: June 10, 2019; Accepted: June 25, 2019; Published: June 28, 2019 


\section{Materials and methods}

The ceramic laminate veneers included in the study were completed by one operator (the researcher) and one experienced dental technician. The patients were randomly divided into 2 equal groups (fourteen laminate veneers for each group) using random sequence generator from the website (www.random.org).

The investigators and the statistician were blind throughout the whole procedures and follow-up visits.

Their chief complaint was to enhance their smile. The treatment plan was explained for each patient. Then, they agreed to sign the informed consent before proceeding to clinical work.

Patients selected were characterized as follows:

1. From 18-50 years old, be able to read and sign the informed consent document.

2. Be physically and psychologically able to tolerate conventional restorative procedures and have no active periodontal or pulpal diseases, have teeth with good restorations.

Scaling and polishing were performed for each patient and shade of the teeth was recorded visually using vita 3D master shade guide under natural day light between 10 am and 12 noon on days with a clear sky.

Preliminary impressions were made to obtain study casts. Following a careful analysis of the models, diagnostic wax-up was fabricated in order to establish the appropriated mock up for the patients.

\section{Preparation}

The labial reduction was started with horizontal orientation grooves in two different planes using depth cutter wheel within range of $0.3-0.5$ $\mathrm{mm}$ at the cervical third and $0.6-0.8 \mathrm{~mm}$ at the middle and incisal thirds to ensure that the whole preparation remained within the enamel. The preparation was ended labially by a chamfer finish line $0.5 \mathrm{~mm}$ diameter equigingivally using a tapered diamond stone with rounded end. Vertical orientation grooves were done on the incisal edge of the teeth using tapered diamond stone of $2 \mathrm{~mm}$ diameter resulting in $2 \mathrm{~mm}$ butt joint incisal preparation. Then preparation was verified with the silicon index to check the amount of incisal and labial reduction.

\section{Final impression}

Putty/light two-step impression was made using addition elastomeric silicon impression material (Express $^{\mathrm{Tn}}$ VPS Impression Material, 3M ESPE, St. Paul, MN, USA) using stock tray.

\section{Provisional restoration}

Silicon index obtained from the waxed up cast was used to construct provisional restoration. It was filled with temporary crown material (Protemp $^{\text {tis }}$ II 3M ESPE, St. Paul, MN, USA) and placed on the teeth that were spot etched and bonded.

\section{Veneer fabrication}

An intra-oral scanner (CEREC Omnicam, Sirona Dental CAD/ CAM System, $\mathrm{GmbH}$ ) was used to scan both master and opposing casts to obtain a virtual model on the computer screen. CEREC premium 4.4 CAD/CAM software was used to prepare the best suitable design for the laminate veneers and the different tools were used to customize each veneer to the final desired shape. The final restorations were tested before their milling by CAD/CAM wax milled with the final shape of the final restorations.
IPS e.max CAD veneers were fired in a firing cycle in a ceramic furnace. Lava Ultimate laminates were polished after milling with fine rubber tool and polishing agent until the luster surface appeared.

\section{Cementation procedures}

\section{Pre-treatment of lava ultimate veneers}

Lava Ultimate veneers were cleaned in an ultrasonic cleaner and gently air blowed. Then, air abraded following the manufacturer instructions to obtain matt surface. The adhesive (Scotchbond ${ }^{m}$ Universal Adhesive, 3M ESPE, St. Paul, MN, USA) was applied to the bonding surface of the veneer and left for 60 seconds to react.

\section{Pre-Treatment of IPS e.max CAD veneers}

IPS e.max CAD veneers were cleaned with alcohol, rinsed with water and air dried for preparing their fitting surface to be etched for 20 seconds with 9\% hydrofluoric acid ( Porcelain Etchant 9\%, BISCO, USA ).The veneers were rinsed with water and dried with air. A single coat of the ceramic primer (Bisco Porcelain Prime/Bis-Silane, BISCO, USA) was then applied to the bonding surface of the veneers and left for 1 minute to react then dried with air.

$37 \%$ Phosphoric acid etchant was applied to enamel for 15 seconds. Then rinsed and dried resulted in frosty enamel appearance. Two coats of adhesive were applied to the teeth surface. The veneers were cemented in place with light cured veneer resin cement (RelyX ${ }^{\mathrm{nt}}$ Veneer Cement | 3M ESPE, St. Paul, MN, USA) using an energy density of 480 $\mathrm{mW} \backslash \mathrm{cm}$ for 40 seconds from each aspect of the tooth. Preoperative and postoperative photographs are shown in figure 1.

\section{Clinical evaluation}

The restorations were visually inspected according to the modified United States Public Health Service (USPHS) criteria with dental mirror and explorer under magnification of dental loupes (Univet 3.5x, Italy). Digital photographs were taken after placement of the veneers of both groups and during follow-up sessions through the whole study period. During the follow up period, no attrition was recorded (Table 1).

Patient satisfaction was evaluated using questionnaire that is shown in figure 2 . The questionnaire was carried out by visual analogue scale (VAS).

\section{Results}

Qualitative data were presented as frequencies and percentages. Fisher's Exact test was used to compare between the two groups. Friedman's test was used to study the changes by time in each group. Patient satisfaction scores (VAS scores) were presented as median and range values. Mann-Whitney $U$ test was used to compare between the two groups. The significance level was set at $\mathrm{P} \leq 0.05$. Statistical analysis was performed with IBM SPSS Statistics Version 20 for Windows.

\section{Marginal adaptation, retention and fracture}

Through the whole study period; all restorations in the two groups showed (Alpha) scores.
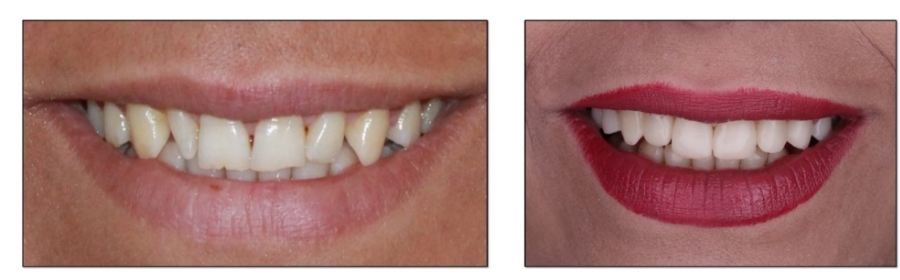

Figure 1. Preoperative and postoperative lava ultimate veneers close up smile view 
Code no. :

Age:

Sex:

Volunteer accepts all procedures of the study and accepts to answer the questionnaire.

You score degrees of satisfy of restorations related to that topic. You should give a mark on the line as shown below.

1-How much are you satisfied about the shape of your teeth?

Baseline

One year recall
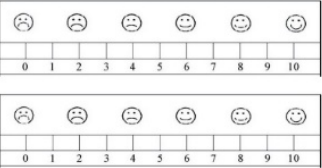

2-How much are you satisfied about the size of your teeth?

Baseline

One year recall

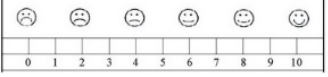

3-How much are you satisfied about the color of your teeth?

Baseline

One year recall
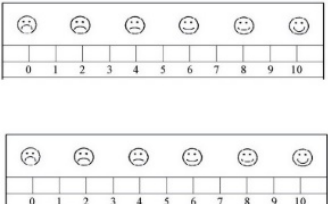

4- Do you have any problems with bleeding gums during brushing the veneer restoration?

Baseline

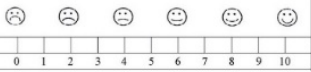

One year recall

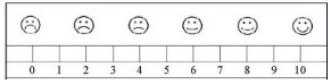

5- Do you have any problems while cleaning the restoration with a toothbrush and/or dental floss after veneer placement?

Baseline

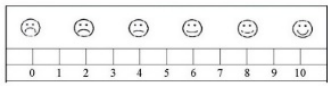

One year recall

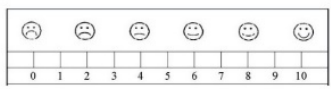

Figure 2. List of the questionnaire for patient satisfaction

Table 1. List of modified United States Public Health Service criteria used for the clinical evaluations of the restorations

\begin{tabular}{|l|l|}
\hline $\begin{array}{l}\text { Marginal } \\
\text { adaptation }\end{array}$ & $\begin{array}{l}\text { A. (Alpha) Smooth margin } \\
\text { B. (Beta) Minor voids/defects at margin (probe catch) } \\
\text { C. (Charlie) Obvious crevice at margin (probe penetrate) }\end{array}$ \\
\hline $\begin{array}{l}\text { Fracture of } \\
\text { restoration }\end{array}$ & $\begin{array}{l}\text { A. No fracture } \\
\text { B. Minor chipping of restoration (1/4 of restoration) } \\
\text { C. Moderate chipping of restoration (1/2 of restoration) } \\
\text { D. Severe chipping of restoration (3/4 of restoration) }\end{array}$ \\
\hline Retention & $\begin{array}{l}\text { A. Retained } \\
\text { B. Debonded }\end{array}$ \\
\hline $\begin{array}{l}\text { Marginal } \\
\text { discoloration }\end{array}$ & $\begin{array}{l}\text { A. No discoloration } \\
\text { B. Obight staining can be polished away }\end{array}$ \\
\hline
\end{tabular}

Where: A: Alpha B: Bravo C: Charlie D: Delta

Alpha and Bravo: satisfactory; Charlie and Delta: unsatisfactory except for retention where Alpha is satisfactory and Bravo is unsatisfactory.

\section{Marginal discoloration}

All surfaces of restorations in the two groups showed (Alpha) scores till 6 months. While after 8,10 and 12 months; the Palatal surface of Lava Ultimate group showed (Bravo) scores. After 8 months, there was no statistically significant difference between the two groups ( $P$-value $=0.481$, Effect size $=0.277)$. After 10 and 12 months, there was also no statistically significant difference between the two groups $(P$-value $=$ 0.098 , Effect size $=0.408$ ) table 2 .

Results of the changes by time within Lava Ultimate group are presented in table 3 . There was a statistically significant change in marginal discoloration by time $(P$ - value $=0.002$, Effect size $=$ 0.494). After 8 months, there was an increase in prevalence of (Bravo) score. After 10 and 12 months, further increase in (Bravo) scores was observed.

\section{Patient satisfaction:}

It was divided into esthetical satisfaction evaluation consisted of shape, size, and color satisfaction. In addition, Biological satisfaction consisted of gingival bleeding and flossing problems. The overall satisfaction of restorations was Very satisfied $85.7 \%$ and Satisfied $14.3 \%$

At base line as well as after 12 months; Lava Ultimate showed statistically significantly higher median shape and lower median color 
ElGendi MM (2019) One-year clinical evaluation of e.max CAD and LavaTM ultimate laminate veneers with butt joint preparation (Randomized controlled clinical trial)

Table 2. Descriptive statistics and results of Fisher's Exact test for comparison between marginal discoloration at the Palatal surfaces of the two groups after 8 , 10 and 12 months

\begin{tabular}{|c|c|c|c|c|c|c|}
\hline \multirow[t]{2}{*}{ Time } & \multicolumn{2}{|c|}{$\begin{array}{l}\text { Lava Ultimate } \\
\quad(n=14)\end{array}$} & \multicolumn{2}{|c|}{$\begin{array}{l}\text { IPS e.max CAD } \\
(n=14)\end{array}$} & \multirow[t]{2}{*}{$P$-value } & \multirow[t]{2}{*}{ Effect size (Cramer's V) } \\
\hline & $\mathbf{n}$ & $\%$ & $\mathbf{n}$ & $\%$ & & \\
\hline 8 months & & & & & \multirow{3}{*}{0.481} & \multirow{3}{*}{0.277} \\
\hline Alpha & 12 & 85.7 & 14 & 100 & & \\
\hline Bravo & 2 & 14.3 & $\mathbf{0}$ & $\mathbf{0}$ & & \\
\hline \multicolumn{5}{|c|}{10 months } & \multirow{3}{*}{0.098} & \multirow{3}{*}{0.408} \\
\hline Alpha & 10 & 71.4 & 14 & 100 & & \\
\hline Bravo & 4 & 28.6 & $\mathbf{0}$ & $\mathbf{0}$ & & \\
\hline \multicolumn{5}{|c|}{12 months } & \multirow{3}{*}{0.098} & \multirow{3}{*}{0.408} \\
\hline Alpha & 10 & 71.4 & 14 & 100 & & \\
\hline Bravo & 4 & 28.6 & $\mathbf{0}$ & $\mathbf{0}$ & & \\
\hline
\end{tabular}

*: Significant at $P \leq 0.05$

Table 3. Descriptive statistics and results of Friedman's test for comparison between marginal discoloration at different follow up periods within Lava Ultimate group

\begin{tabular}{|c|c|c|}
\hline \multirow[t]{2}{*}{ Time } & \multicolumn{2}{|c|}{$\begin{array}{l}\text { Lava Ultimate } \\
\quad(n=14)\end{array}$} \\
\hline & $\mathbf{N}$ & $\%$ \\
\hline \multicolumn{3}{|l|}{ Base line } \\
\hline Alpha & 14 & 100 \\
\hline Bravo & $\mathbf{0}$ & $\mathbf{0}$ \\
\hline \multicolumn{3}{|l|}{2 months } \\
\hline Alpha & 14 & 100 \\
\hline Bravo & $\mathbf{0}$ & $\mathbf{0}$ \\
\hline \multicolumn{3}{|l|}{4 months } \\
\hline Alpha & 14 & 100 \\
\hline Bravo & $\mathbf{0}$ & $\mathbf{0}$ \\
\hline \multicolumn{3}{|l|}{6 months } \\
\hline Alpha & 14 & 100 \\
\hline Bravo & $\mathbf{0}$ & $\mathbf{0}$ \\
\hline \multicolumn{3}{|l|}{8 months } \\
\hline Alpha & 12 & 85.7 \\
\hline Bravo & 2 & 14.3 \\
\hline \multicolumn{3}{|l|}{10 months } \\
\hline Alpha & 10 & 71.4 \\
\hline Bravo & 4 & 28.6 \\
\hline \multicolumn{3}{|l|}{12 months } \\
\hline Alpha & 10 & 71.4 \\
\hline Bravo & 4 & 28.6 \\
\hline$P$-value & \multicolumn{2}{|c|}{$0.002 *$} \\
\hline Effect size (w) & & \\
\hline
\end{tabular}

*: Significant at $P \leq 0.05$

Table 4. The median, range values and results of Mann-Whitney U test for comparison between patient 5satisfaction (VAS scores) in the two groups

\begin{tabular}{|c|c|c|c|c|c|c|c|c|c|}
\hline \multirow{2}{*}{ Time } & \multirow{2}{*}{ Item } & \multicolumn{3}{|c|}{ Lava Ultimate } & \multicolumn{3}{|c|}{ IPS e.max CAD } & \multirow{2}{*}{$P$-value } & \multirow{2}{*}{ Effect size ( $r$ ) } \\
\hline & & Median & Min. & Max. & Median & Min. & Max. & & \\
\hline \multirow{5}{*}{ Base line } & Shape & 10 & 9 & 10 & 9 & 9 & 10 & $0.026^{*}$ & 0.421 \\
\hline & Size & 10 & 10 & 10 & 10 & 10 & 10 & 1.000 & 0.000 \\
\hline & Color & 9 & 9 & 10 & 10 & 9 & 10 & $0.026^{*}$ & 0.421 \\
\hline & Gingival bleeding & 10 & 10 & 10 & 10 & 10 & 10 & 1.000 & 0.000 \\
\hline & Flossing & 10 & 10 & 10 & 10 & 10 & 10 & 1.000 & 0.000 \\
\hline \multirow{5}{*}{12 months } & Shape & 10 & 9 & 10 & 9 & 9 & 10 & $0.026^{*}$ & 0.421 \\
\hline & Size & 10 & 10 & 10 & 10 & 10 & 10 & 1.000 & 0.000 \\
\hline & Color & 9 & 9 & 10 & 10 & 9 & 10 & $0.026^{*}$ & 0.421 \\
\hline & $\begin{array}{l}\text { Gingival } \\
\text { bleeding }\end{array}$ & 10 & 10 & 10 & 10 & 7 & 10 & 0.056 & 0.509 \\
\hline & Flossing & 10 & 10 & 10 & 10 & 10 & 10 & 1.000 & 0.000 \\
\hline
\end{tabular}

*: Significant at $P \leq 0.05$ 
scores than e.max. There was no statistically significant difference between size, gingival bleeding and flossing scores in the two groups table 4.

\section{Discussion}

In comparison to full coverage crowns, ceramic laminate veneers are minimal invasive treatment method in restorative dentistry and can be used to correct tooth form, position and color [5].

The outcomes recorded (marginal adaptation, biocompatibility, esthetics and mechanical strength) are considered distinct properties for evaluating the clinical success of dental restoration [6].

Conventional and current popular practice for conservative laminate veneers preparation on the facial surface, the reduction ranged from 0.3 to $0.8 \mathrm{~mm}$ and on the incisal edge from 1.5 to $2 \mathrm{~mm}$ that was suggested by many studies $[7,8]$.

In the present study, preparation design for all teeth was butt-joint that was followed by many other authors who reported that butt-joint preparation showed better stress distribution to the tooth body and highest fracture resistance compared with the other techniques $[9,10]$.

In the present study, Lava Ultimate CAD/CAM veneers were sandblasted with aluminum oxide grain size $\leq 50 \mu \mathrm{m}$ at two bars (30 psi) for $15 \mathrm{sec}$ to increase the surface roughness and result in an irregular surface with some fillers exposure. But Yoshihara et al, 2017 [11] stated that sandblasting also resulted in damaging the composite $\mathrm{CAD} / \mathrm{CAM}$ block surface as it produce surface and subsurface cracks.

Therefore, sandblasting for CAD/CAM resin blocks must be mild by adjusting both grain size and time as suggested by Tekçe et al. 2018 [12] not to exceed 30 seconds of sandblasting for CAD/CAM resin restoratives as by increasing sandblasting time, surface roughness increased, however, $\mu$ TBS values decreased and stability of adhesion of $\mathrm{CAD} / \mathrm{CAM}$ composite restoratives would be disturbed.

In the present study, Scotchbond ${ }^{\mathrm{mi}}$ Universal Adhesive containing silane coupling agent was applied to the bonding surface of the Lava Ultimate veneers and scrubbed them for 20 seconds then left for 60 seconds to react that was in consistent with Capa et al. 2018 [13] who proved that following sandblasting, chemical adhesion using a universal adhesive containing silane was effective surface pre-treatment methods for resin nano ceramic CAD/CAM.

The clinical evaluation parameters in this study were following the modified USPHS (Modified United States Public Health Service Criteria). Most studies had followed the modified USPHS/Ryge Criteria for direct clinical evaluation of restoration due to its simplicity to evaluate the clinical veneers and analyze the results enabling the assessment of multiple parameters and provide reliable information regarding the overall long-term success of the restorations [14].

\section{Marginal adaptation}

The results obtained in the present study as alpha score for marginal adaptation may be attributed to the butt-joint incisal configuration and preservation of a peripheral enamel layer around all margins, this design established an easy path of insertion of the laminate during bonding [15]. Our results were similar to other authors who found that the $\mathrm{CAD} / \mathrm{C} / \mathrm{AM}$ veneers with butt joint incisal reduction produced the most accurate margins with gap $<100 \mu \mathrm{m}$ in the cervical, middle, and incisal thirds. While the least favorable combination was the pressable ceramic veneers with overlapped incisal reduction [16]. In addition, resin cement used might be effectively reduced the micro-leakage [17].
Following total-etch system for bonding ceramic laminate veneers might be also a reason for successful marginal adaptation and detection of no secondary caries [18].

\section{Fracture}

In this study, alpha score was recorded for all veneers during all the follow-up sessions that run similar to other studies [19].

\section{Retention}

The alpha score of retained/debonding was recorded for all veneers of both groups. All the teeth was prepared and finished with margins located within enamel, which explained the results of this study that was in agreement with Ozturk et al. 2014 [20] who showed that porcelain veneers partially bonded to dentin, had an increased risk of failure.

\section{Marginal discoloration:}

Labially, all the veneers scored Alpha for all the follow up periods which can be attributed to many reasons: (1) All margins are in cleansable areas often easily finished and polished at the time of cementation [21], (2) the glazed surface of IPS e.max CAD [22], and properly finished and polished surface of Lava Ultimate which is mostly impervious to extrinsic stains [23], that protects the underlying light-cured (more color stable) resin cement [24]; and (3) well-fitted restorations and a thin viscosity, but highly filled, resin cement have been used [25].

Palatally, four Lava Ultimate veneers scored Bravo after 12 months follow up period where marginal staining were mainly observed at the incisal edges only from the lingual aspect. Since in these regions, function play a significant role compared to labial surfaces, aging of the adhesive resin or the luting cement, cement washout or initial polymerization shrinkage may be responsible from such deteriorations and eventually staining [26].

The clinical outcome showed marginal discoloration in $14.3 \%$ and $28.6 \%$ of all the restorations and Lava Ultimate restorations respectively. This result is comparable to other studies that found marginal discoloration in $21.3 \%$ of restorations [5] but it is considered higher percentage than Monaraks and Leevailoj 2018 [14] who showed low rate of marginal discoloration $(0.6 \%)$, found in the veneer restoration of a maxillary central incisor at the palatal site.

The statistical significant change in marginal discoloration of the Lava Ultimate group by time was considered clinically acceptable as they went unnoticed by the patients and once detected it could be easily repolished. Such findings require longer follow up period to assess whether repolishing of the discoloration would still be effective.

\section{Patient satisfaction}

In the majority of the clinical studies on this type of restorations, the degree of patient satisfaction has been a factor taken into account. Although Lava Ultimate showed statistically significantly higher median shape and lower median color scores than IPS e.max CAD for patient satisfaction, it was considered clinically insignificant as the patients rated their shape and color satisfaction as 'very satisfied' with score ranging from 9 to 10 . The percentage of laminates for the two groups, which reported being 'very satisfied' with score (8-10) and 'satisfied' with score (7) were $85.7 \%$ and $14.3 \%$ respectively. Our study revealed results that were very similar to those found by other authors. $[5,6,14]$ Patient satisfaction was encouraging, even in patients who recorded low scores.

The hypothesis of this study was accepted since the descriptive data of the two groups in terms of retention, marginal adaptation and 
fracture showed no difference (Alpha score). In addition, the difference between the two groups in marginal discoloration was not statistically significant. Although the recorded scores of patient satisfaction were statistically significant between the two groups regarding 'shape' and 'color', they reported no clinical significant difference.

\section{Conclusion}

Within the limitations of this study, it can be concluded that both IPS e.max CAD and Lava Ultimate laminate veneers revealed high successful clinical performance in terms of marginal adaptation, fracture, retention, marginal discoloration and patient satisfaction. It can be recommended to implement further randomized clinical studies to evaluate the clinical survival rate of the Lava ${ }^{\mathrm{TM}}$ Ultimate laminate veneers over a more prolonged follow up periods.

\section{References}

1. Pimentel W, Teixeira ML, Costa PP, Jorge MZ, Tiossi R (2016) Predictable Outcomes with Porcelain Laminate Veneers: A Clinical Report. J Prosthodont 25: 335-340. [Crossref]

2. Jha R, Jain V, Das TK, Shah N, Pruthi G (2013) Comparison of Marginal Fidelity and Surface Roughness of Porcelain Veneers Fabricated by Refractory Die and Pressing Techniques. J Prosthodont 22: 439-44. [Crossref]

3. Ruse ND, Sadoun MJ (2014) Resin-composite blocks for dental CAD/CAM applications. J Dent Res 93: 1232-1234. [Crossref]

4. Kang S, Chang J, Son H (2013) Flexural strength and microstructure of two lithium disilicate glass ceramics for CAD/CAM restoration in the dental clinic. Restor. Restor Dent Endod 38: 134-140. [Crossref]

5. Beier US, Kapferer I, Burtscher D, Dumfahrt H (2012) Clinical performance of porcelain laminate veneers for up to 20 years. Int J Prosthodont 25: 79-85. [Crossref]

6. Nejatidanesh F, Savabi G, Amjadi M, Abbasi M, Savabi O (2018) Five year clinical outcomes and survival of chairside $\mathrm{CAD} / \mathrm{CAM}$ ceramic laminate veneers a retrospective study. J Prosthodont Res 62: 462-467, 2018. [Crossref]

7. Radz GM (2011) Minimum thickness anterior porcelain restorations. Dent Clin North Am 55: 353-370, 2011. [Crossref]

8. Costa DC, Coutinho M, de Sousa AS, Ennes JP (2013) A Meta-Analysis of the Most Indicated Preparation Design for Porcelain Laminate Veneers. J Adhes Dent 15: 215 220. [Crossref]

9. Tugcu E, Vanlioglu B, Özkan YK, Aslan YU (2018) Marginal Adaptation and Fracture Resistance of Lithium Disilicate Laminate Veneers on Teeth with Different Preparation Depths. Int J Periodontics Restorative Dent 38: s87-s95. [Crossref]

10. Ustun O, Ozturk AN (2018) The Evaluation of Stress Patterns in Porcelain Laminate Veneers with Different Restoration Designs and Loading Angles Induced by Functional Loads: A Three-dimensional Finite Element Analysis Study. Niger J Clin Pract 21: 337-334. [Crossref]
11. Yoshihara K, Nagaoka N, Maruo Y, Nishigawa G, Irie M, et al. (2017) Sandblasting may damage the surface of composite CAD-CAM blocks. Dent Mater 33: e124124e135. [Crossref]

12. Tekçe N, Tuncer S, Demirci M (2018) The effect of sandblasting duration on the bond durability of dualcure adhesive cement to CAD/CAM resin restorative. $J A d v$ Prosthodont 10: 211-217. [Crossref]

13. Capa N, Say EC, Celebi C, Casur A (2019) Microtensile bond strengths of adhesively bonded polymer-based CAD/CAM materials to dentin. Dent Mater J 38: 75-85. [Crossref]

14. Monaraks R, Leevailoj C (2018) The Longevity of Ceramic Veneers: Clinical Evaluation of Mechanical, Biologic and Aesthetic Performances of Ceramic Veneers, a 7-year Retrospective Study. J DENT ASSOC THAI 68: 288-301.

15. Najim BA, Al-Rawi II (2015) The Influence of Different Fabrication Techniques and Preparation Designs on the Marginal Adaptation of Ceramic Veneers? An in Vitro Comparative Study. J Baghdad Coll Dent 27: 8-14.

16. Pereira DD, Marquezan M, Grossi ML, Silva Oshima HM (2018) Analysis of Margina Adaptation of porcelain Laminate Veneers Produced by Computer-Aided Design/ Computer-Assisted Manufacturing Technology: A Preliminary In Vitro Study. Int $J$ Prosthodont 31: 346-348. [Crossref]

17. Medic V, Obradovic-Djuricic K, Dodic S, Petrovic R (2010) In Vitro evaluation of microleakage of various types of dental cements. Srp Arh Celok Lek 138: 143-149. [Crossref]

18. Aykor A, Ozel E (2009) Five-year Clinical Evaluation of 300 Teeth Restored with Porcelain Laminate Veneers Using Total-etch and a Modified Self-etch Adhesive System. Oper Dent 34: 516-523. [Crossref]

19. Zierden K, Acar J, Rehmann P, Wostmann B (2018) Wear and Fracture Strength of New Ceramic Resins for Chairside Milling. Int J Prosthodont 31: 74-76. [Crossref]

20. Öztürk E, Bolay S (2014) Survival of porcelain laminate veneers with different degrees of dentin exposure: 2-year clinical results. J Adhes Dent 16: 481-489. [Crossref]

21. Gurel G, Sesma N, Calamita MA, Coachman C, Morimoto S (2013) Influence of enamel preservation on failure rates of porcelain laminate veneers. Int J Periodontics Restorative Dent 33: 31-39. [Crossref]

22. Palla ES, Kontonasaki E, Kantiranis N, Papadopoulou L, Zorba T, et al. (2018) Color stability of lithium disilicate ceramics after aging and immersion in common beverages. J Prosthet Dent 119: 632-642. [Crossref]

23. Quek SHQ, Yap AUJ, Rosa V, Tan KBC, Teoh KH (2018) Effect of staining beverages on color and translucency of CAD/CAM composites. J Esthet Restor Dent 30: E99E17. [Crossref]

24. Marchionatti AME, Wandscher VF, May MM, Bottino MA, May LG (2017) Color stability of ceramic laminate veneers cemented with light-polymerizing and dualpolymerizing luting agent: A split-mouth randomized clinical trial. J Prosthet Dent 118: 604-610. [Crossref]

25. Calamia JR, Calamia CS (2007) Porcelain laminate veneers: reasons for 25 years of success. Dent Clin North Am 51: 399-417, ix. [Crossref]

26. Gresnigt MM1, Kalk W, Özcan M (2013) Clinical longevity of ceramic laminate veneers bonded to teeth with and without existing composite restorations up to 40 months. Clin Oral Investig 17: 823-832. [Crossref]

Copyright: (C2019 ElGendi MM. This is an open-access article distributed under the terms of the Creative Commons Attribution License, which permits unrestricted use, distribution, and reproduction in any medium, provided the original author and source are credited. 